\title{
Extraabdominal parasitic lipoleiomyoma
}

\author{
Tae Hoon Lee, Se-Jin Baek \\ Department of Surgery, Korea University College of Medicine, Seoul, Korea
}

Extrauterine parasitic lipoleiomyoma is a very rare fatty tumor, with uncertain histopathogenesis. Although imaging studies play an important role in preoperative localization and diagnosis of lipoleiomyoma, a pathological evaluation is paramount for confirmation of diagnosis. We describe a case of a 49-year-old woman with a palpable mass in the right inguinal area. Computed tomography of the abdomen and pelvis revealed a fluid- and fat-containing mass. Histopathological examination of the mass, which was successfully resected, confirmed the diagnosis of lipoleiomyoma. The patient was discharged on a postoperative day 2 without any complications.

Keywords: Extraabdominal neoplasm, Extrauterine neoplasm, Lipoleiomyoma, Lipoma, Spindle cell tumor

\section{INTRODUCTION}

Lipoleiomyoma is an uncommon benign neoplasm containing adipose cells, hyperplastic smooth muscle, and fibrous tissue, and it is typically well encapsulated. This tumor is considered a variant of lipomatous lesions, and its incidence ranges from $0.03 \%$ to $0.20 \%$ [1]. Lipoleiomyoma is most often observed in the uterus, and the clinical manifestations include pelvic discomfort and menstrual disturbances [2]. Imaging studies such as abdominopelvic computed tomography (CT) and pelvic magnetic resonance imaging (MRI) can play an important role in diagnosing a lipoleiomyoma, but pathologic evaluation must be performed to confirm the diagnosis ultimately. Although the etiology is unclear, the tumor has been considered benign and the treatment options range from observation to surgical treatment. Compared to uterine lipoleiomyoma, extrauterine lipoleiomyoma is rare, and of these, extraabdominal-cum-extrauterine lipoleiomyoma has been extremely rarely reported. In this report, we present our experience with a

Received: Apr 29, 2021 Revised: Jun 10, 2021 Accepted: Jun 14, 2021

Correspondence to: Se-Jin Baek

Department of Surgery, Korea University College of Medicine,

73 Goryeodae-ro, Seongbuk-gu, Seoul 02841, Korea

Tel: +82-2-920-6412, Fax: +82-2-928-1631

E-mail:xezin@korea.ac.kr

ORCID: Tae Hoon Lee (https://orcid.org/0000-0002-9700-9479), Se-Jin Baek (https://orcid.org/0000-0002-3185-8777)

Copyright (C) 2021 Korean Society of Surgical Oncology

This is an Open Access article distributed under the terms of the Creative Commons Attribution Non-Commercial License (http://creativecommons.org/licenses/by-nc/4.0) which permits unrestricted non-commercial use, distribution, and reproduction in any medium, provided the original work is properly cited. successful excision of a large, extraabdominal, extrauterine lipoleiomyoma located in the inguinal area. This study was approved by the Institutional Review Board of Korea University Anam Hospital (IRB No. 2020AN0479) and the written informed consent was obtained.

\section{CASE REPORT}

A 49-year-old female patient presented to the outpatient clinic with a 6-month history of a palpable mass in the right inguinal area. The patient had undergone two cesarean sections and had no underlying diseases other than hypertension. On physical examination, the mass was painless, fist-sized, and round-shaped which unchanged with posture. It was not manually reducible.

To differentiate a sliding inguinal hernia of an internal organ, such as an ovary, the patient underwent abdominopelvic CT scan, which revealed an elongated lesion with a long diameter of about $11 \mathrm{~cm}$ from the right inguinal area to the right vulvar area (Fig. 1). Inside the lesion, multiple ovoid enhancing nodules were observed with fluid and fat attenuations. There were no abnormalities found in the uterus and bilateral adnexa and ovaries.

Elective surgery under general anesthesia was performed with the patient positioned supine. First, we performed an intraperitoneal laparoscopic exploration to exclude herniation of any intraabdominal organs, including the ovaries. A 12-mm trocar was inserted into the umbilicus for the camera, and two 5-mm trocars were inserted into the left middle and lower quadrants. After a normal laparoscopy of the abdominal cavity, we made a $7 \mathrm{~cm}$ incision over the mass and meticulously dissected around the mass. The planes were easily defined, and the well-encapsulated mass was resected 
as a whole without damage. The operation time was around 140 minutes. The patient started oral feeding 6 hours after surgery and was discharged without complications on the 2nd day after surgery. At the 1-week outpatient follow-up visit, the patient had no postoperative complications and no recurrence was observed after 1 year of follow-up.

On gross pathologic examination of the specimen, the tumor
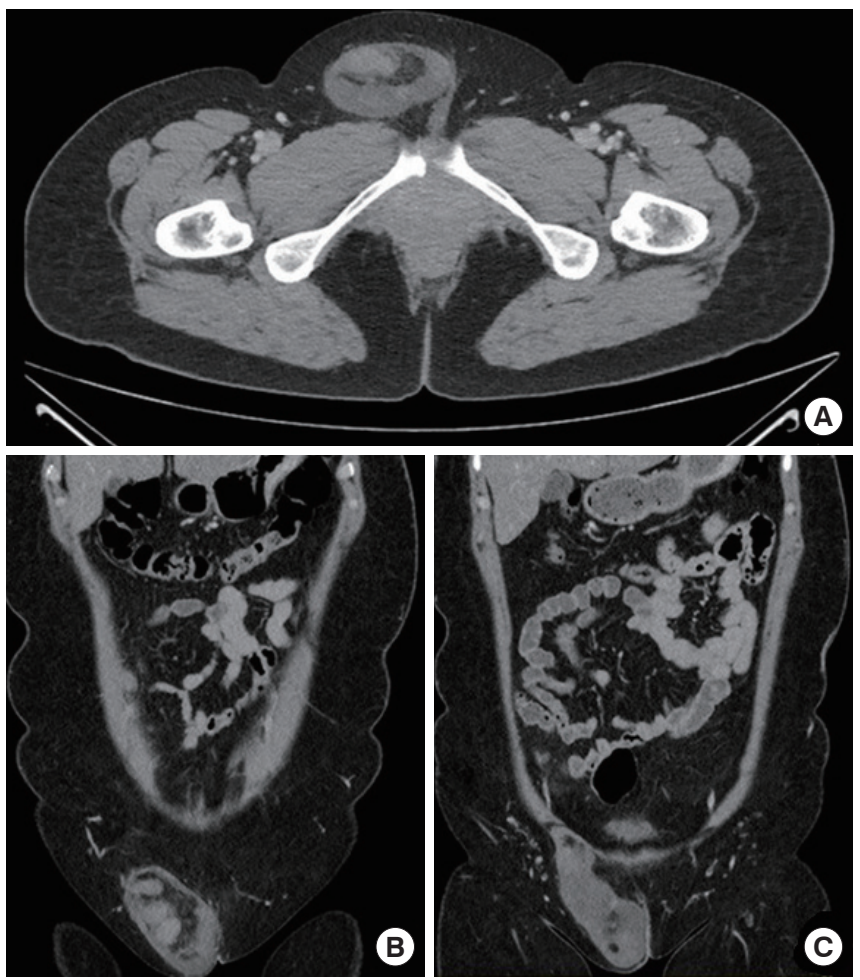

Fig. 1. Abdominopelvic computed tomography scan showing a tumor containing ovoid enhancing nodules and a fat-with-fluid collection. (A) Axial view. $(B, C)$ Coronal view. The tumor spread from the right inguinal area to the right vulvar area (C).
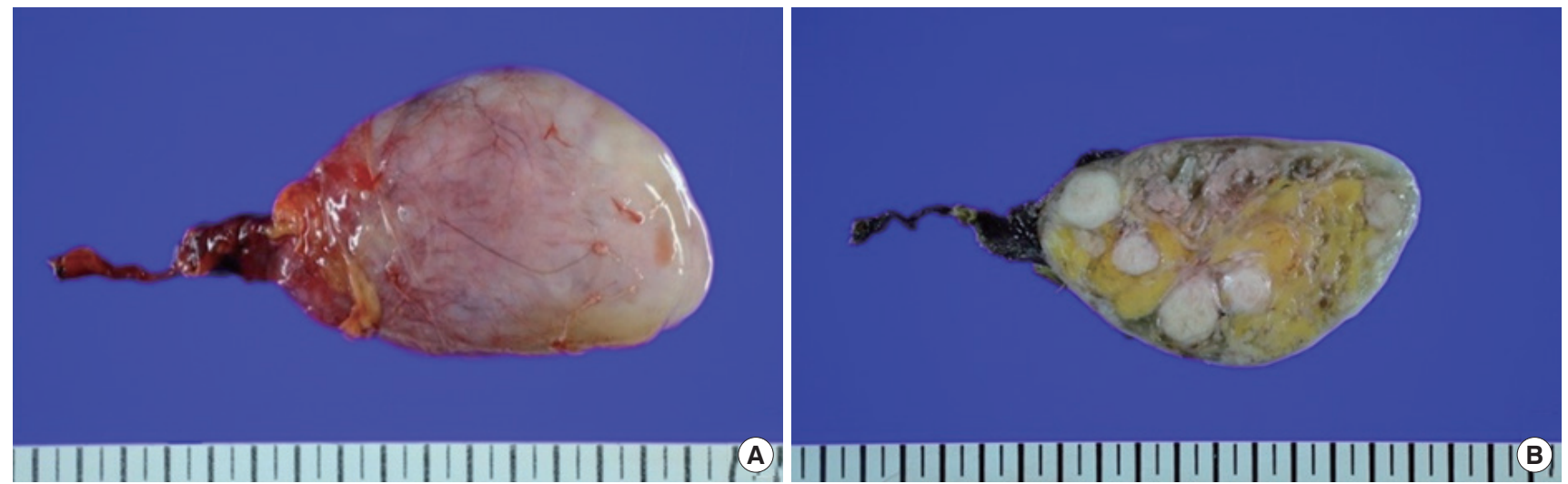

Fig. 2. Gross findings of the resected specimen. (A) A grayish, ovoid mass measured $12.0 \times 6.0 \times 3.5 \mathrm{~cm}$. (B) The cut surface shows yellowish fatty portions, grayish solid whirling portions, and vessel-like portions.

measured $12.0 \times 6.0 \mathrm{~cm}$ (Fig. 2A). It showed different areas on its cut surface; there were yellowish fatty portions, grayish solid whirling portions, and vessel-like portions (Fig. 2B). The tumor was composed of mature adipocytes with smooth muscle cells (Fig. 3). Immunohistochemical analyses revealed that bundles of spindle cells were positive for desmin and smooth muscle actin (Fig. 4), and the final pathological diagnosis confirmed a lipoleiomyoma.

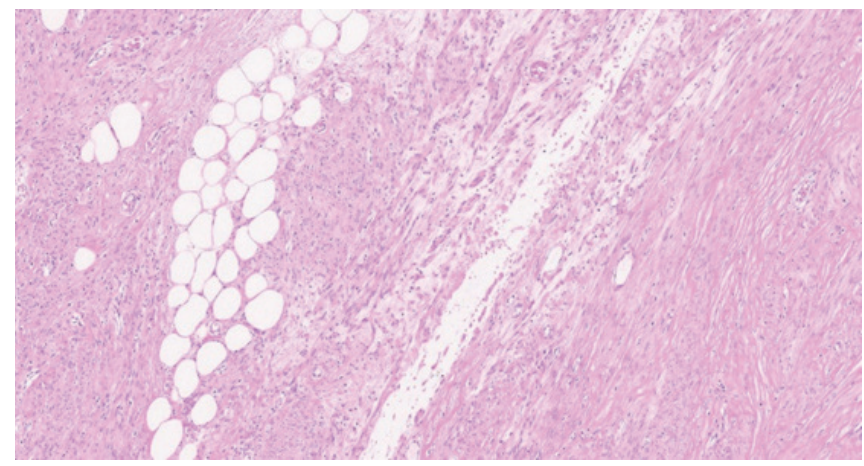

Fig. 3. The tumor was composed of mature adipocytes intermixed with smooth muscle cells $\left(H \& E_{1} \times 50\right)$.

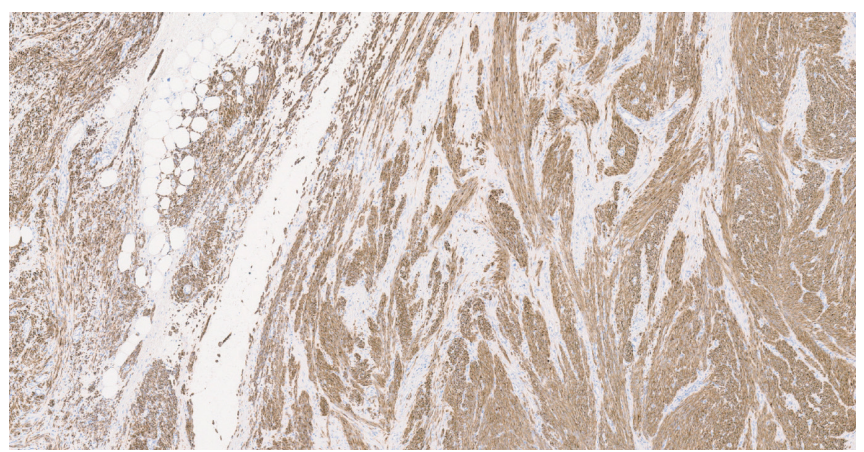

Fig. 4. Immunohistochemical staining $(\times 50)$ demonstrates positivity for desmin and smooth muscle actin. 


\section{DISCUSSION}

Lipoleiomyomas were first described in 1991 by Meis and Enzinger [3] as a myolipoma of the soft tissue. The majority of the tumors are found in the subserosal or intramural uterine corpus, although there have been reported occurrences in the cervix, ovarian areas, broad ligament, and retroperitoneum. In rare cases, extraabdominal lesions have also been reported. Basara Akin et al. [4] reported a lipoleiomyoma in the parasternal area, in the peripheral part of upper inner quadrant of breast, and Vasconcelos et al. [5] reported a large intraperitoneal lipoleiomyoma $(36 \mathrm{~cm})$ connected by a stalk to the parietal peritoneum. To the best of our knowledge, this current case is the first report of an extraabdominal parasitic lipoleiomyoma observed in the inguinal area.

According to previous reports, uterine lipoleiomyomas generally occur in obese perimenopausal or menopausal women $[2,6]$. The patient in our case was a premenopausal woman with a body mass index of $30.16 \mathrm{~kg} / \mathrm{m}^{2}$, categorized as relatively obese in Asian women. Clinical presentation of uterine lipoleiomyomas is usually nonspecific. Most patients are asymptomatic, although some present with abnormal uterine bleeding, menstrual abnormalities, a palpable mass, or abdominal and/or pelvic pain [2]. In the case of an extrauterine lipoleiomyoma, various symptoms, such as abdominal pain and a palpable mass, may appear depending on the location.

Although the pathogenesis remains unclear, immunohistochemical studies suggest that lipoleiomyomas may arise from immature mesenchymal cells or from metaplasia of uterine smooth muscle cells [6]. Akbulut et al. [7] suggested that estrogenic manifestations may be an important factor in development of the tumor; retrospectively analysis of 76 lipoleiomyoma cases with $75.7 \%$ of patients having different types of lesions associated with hyper-estrogenic status, such as adenomyosis, endometriosis, endometrial hyperplasia, polyps, complex atypical endometrial hyperplasia, and gynecologic carcinomas. Lin et al. [8] reported that changes in lipid and other non-lipid metabolism during menopause may be associated with lipoleiomyomas. Furthermore, they may be associated with other metabolic disorders, such as hyperlipidemia, hypothyroidism, and diabetes mellitus.

Although asymptomatic patients require no treatment, these tumors may be confused with ovarian teratoma and liposarcoma, which require surgical excision [6]. Imaging studies such as CT and MRI play an important role in preoperative diagnosis and determination of the exact location of the tumor. Because of the fat component inside the tumor, a high-signal intensity heterogeneous mass can be observed in T1-weighted images on MRI. Although extrauterine lipoleiomyoma is less common, it should be included in the differential diagnosis if a fat-containing mass is observed in a patient of advanced age. Finally, pathological evaluation must be performed to confirm the diagnosis. In general, in immunohistochemical evaluation, diffuse adipocytes and smooth muscle cells are mixed and present in different areas without displaying any cytologic atypia, immature lipoblast, or pleomorphic characteristics.

Lipoleiomyomas are clinically similar to leiomyomas in terms of prognosis, behaving in a benign fashion, and are not associated with recurrence following resection. However, liposarcomas can develop from lipoleiomyoma in rare cases. McDonald et al. [9] reported three cases of liposarcomas arising from uterine lipoleiomyomas. In the histopathologic examinations, they showed cellular pleomorphism, brisk mitotic activity, lipoblasts, and infiltrative margins, which differentiated the masses from lipoleiomyoma. Due to the possibility of liposarcoma transformation, follow-up observation may be necessary for a certain period post excisional surgery.

In conclusion, extraabdominal parasitic lipoleiomyoma is a rare neoplasm, and it may be difficult to differentiate from other diseases depending on the location of occurrence. Preoperative imaging studies can help with diagnosis, and detailed history taking for metabolic syndromes should be conducted.

\section{CONFLICT OF INTEREST}

No potential conflict of interest relevant to this article was reported.

\section{REFERENCES}

1. Manjunatha HK, Ramaswamy AS, Kumar BS, Kumar SP, Krishna L. Lipoleiomyoma of uterus in a postmenopausal woman. J Midlife Health 2010;1:86-8.

2. Oh SR, Cho YJ, Han M, Bae JW, Park JW, Rha SH. Uterine lipoleiomyoma in peri or postmenopausal women. J Menopausal Med 2015;21:165-70.

3. Meis JM, Enzinger FM. Myolipoma of soft tissue. Am J Surg Pathol 1991;15:121-5.

4. Basara Akin I, Guray Durak M, Sevinc AI, Aksoy SO, Cevizci MC, Balci P. Parasternal lipoleiomyoma mimicking breast lesion. Breast J 2020;26:1418-20.

5. Vasconcelos C, Cunha TM, Felix A. Lipoleiomyoma of the peritoneum. Acta Radiol 2007;48:10-2.

6. Wang X, Kumar D, Seidman JD. Uterine lipoleiomyomas: a clinicopathologic study of 50 cases. Int J Gynecol Pathol 2006;25:23942.

7. Akbulut M, Gundogan M, Yorukoglu A. Clinical and pathological 
features of lipoleiomyoma of the uterine corpus: a review of 76 cases. Balkan Med J 2014;31:224-9.

8. Lin KC, Sheu BC, Huang SC. Lipoleiomyoma of the uterus. Int J Gynaecol Obstet 1999;67:47-9.
9. McDonald AG, Dal Cin P, Ganguly A, Campbell S, Imai Y, Rosenberg AE, et al. Liposarcoma arising in uterine lipoleiomyoma: a report of 3 cases and review of the literature. Am J Surg Pathol 2011; 35:221-7. 